\title{
Atomic Resolution Imaging and Spectroscopy of Pt-alloy Electrocatalytic Nanoparticles
}

Sagar Prabhudev, ${ }^{1}$ Matthieu Bugnet, ${ }^{1}$ Cory N.Chiang, ${ }^{1}$ Michael Chatzidakis, ${ }^{1}$ Guo-zhen Zhu, ${ }^{2}$ and Gianluigi A. Botton ${ }^{1}$

\footnotetext{
${ }^{1}$ Department of Materials Science and Engineering, McMaster University, 1280 Main St. W., Hamilton, Canada

${ }^{2}$ School of Materials Science and Engineering, Shanghai Jiao Tong University, Shanghai, 200240, P.R. China
}

Platinum-alloy nanoparticles (Pt-alloy) are a system of great interest for the fuel cell electrocatalysis. The cathodic reaction (called the oxygen reduction reaction (ORR)) in a typical polymer electrolyte membrane (PEM) fuel cell is notoriously sluggish owing to its multi-electron pathway, requiring efficient catalysts to accelerate the kinetics. Traditionally, Pt was being used in this regard, but despite its exceptional catalytic activity it is now widely accepted to be an unrealistic choice due to depleting sources and exceedingly high costs. In an ongoing attempt to reduce the mass loading of $\mathrm{Pt}$, there has been a tremendous research till date, constantly suggesting that a nanoscale alloying of Pt with $3 d$ transition metals (TM) and the Pt group metals (PGM) is a more viable option. Finetuning the surface-structure during synthesis and phase transformations can enhance their catalytic activity and durability by manifolds. However, control and feedback on the synthesis demand characterization techniques that provide atomic-resolution chemical information. The recent developments in electron microscopy pertaining to its instrumentation (aberration correctors, ultrafast electron energy loss spectrometers, monochromators) and the innovation with respect to specimen holders (in situ liquid cell, in situ heating, tomography) have had significant benefits on the study of catalyst nanoparticles by providing chemical analysis and imaging on the atomic-scale [1]. This report describes an overview of recent examples and insightful novel findings related to the study of various catalyst nanoparticles, including Pt-Fe [2], Pt-Au and Pt-Au-Co [3] systems where aberration correction has played a major role in understanding the structures of these materials. Discussed below are two highlights central to our present work.

Thermal annealing is one of the prominent modes of creating Pt-enriched surface structures (Figure 1) in the Pt-alloy systems and it is widely known that the catalytic activity is thereby enhanced due to a compressive strain induced on the Pt-shell [2]. However, the nanoscale phase transformation of an ensemble of alloy nanoparticles, polydispersed in both their size and composition, results in an assortment of materials with miscellaneous properties. Controlling their collective evolution and probing the interplay between compositional segregation and atomic-ordering is traditionally carried out via ex-situ approaches by deriving inference before and after annealing. Given the inherent dynamicity associated with nanoscale processes, an atomic-resolved and dynamic perspective has not been possible so far. Here we demonstrate an in situ atomic-resolution imaging and spectroscopy study carried out on the same Pt-alloy nanoparticles, over the course of thermal treatment [4]. While our STEM-HAADF results clearly demonstrate evolution of particle shape, size, ordering and sintering kinetics over the course of heat treatment, the EELS maps reveal new insights into the segregation (Figure 2) and phase separation (Figure 3) process.

Structural degradation studies can screen various catalyst designs based on their durability and also, provide insights into the degradation mechanism itself. So far, these studies are solely limited to 
interpreting the cyclic voltammograms (during potential cycling). Here, we couple the traditional potential cycling (in a liquid electrolyte) with TEM bright-field imaging [5]. This report describes our recent findings on simultaneous investigation of both structural evolution and electrochemical responses of Pt-Fe nanoalloy catalyst particles, using an in-situ liquid cell inside a TEM. We illustrate how the coarsening mechanisms, including nucleation and growth, are not uniform, both in space and in time scale. For instance, the growth rate was found to be both site- and potentialdependent [6].

\section{References}

[1] J Liu, ChemCatChem, 3 (2011), p. 934

[2] S Prabhudev et al., ACS Nano, 7 (2013), p. 6103.

[3] X Tan et al., ACS Catalysis, 5 (2015), p. 1513.

[4] S Prabhudev et al., (submitted).

[5] G-z Zhu and S Prabhudev (equal contribution) et al., J. Phys. Chem. C, 118 (2014), p. 22111.

[6] This work is supported by the CaRPE-FC network (Automotive Partnership Canada) and the microscopy was carried at the Canadian Centre for Electron Microscopy, a facility supported by NSERC, the Canada Foundation for Innovation and McMaster University.
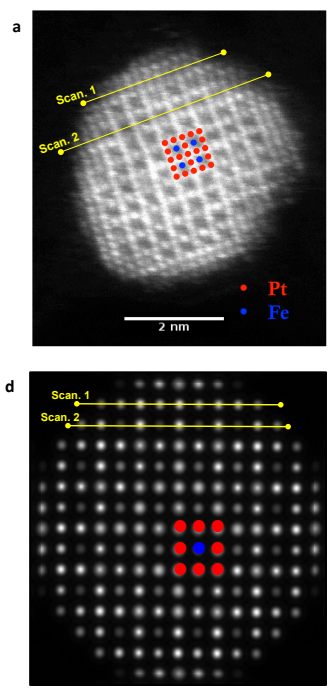

Figure. 1: a, Atomic resolution STEM-HAADF image of a typical Pt-Fe ordered nanoparticle along [010] orientation. d, Multislice simulation of atomic model shown in $\mathbf{c}$. $\mathbf{b}$ and e, Intensity profiles from line scans 1 and 2 corresponding to images in a and d, respectively. f, 2-D surface relaxation mapping on a catalyst nanoparticle. The color bar indicates calculated percentage relaxation with respect to bulk.

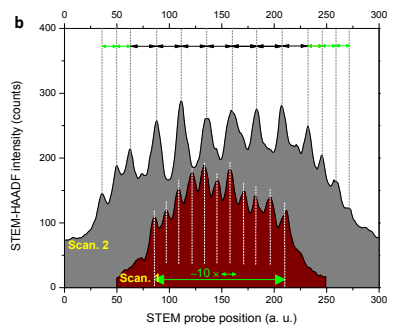

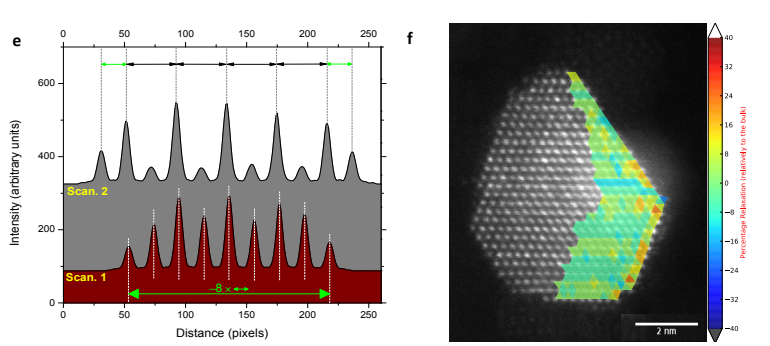
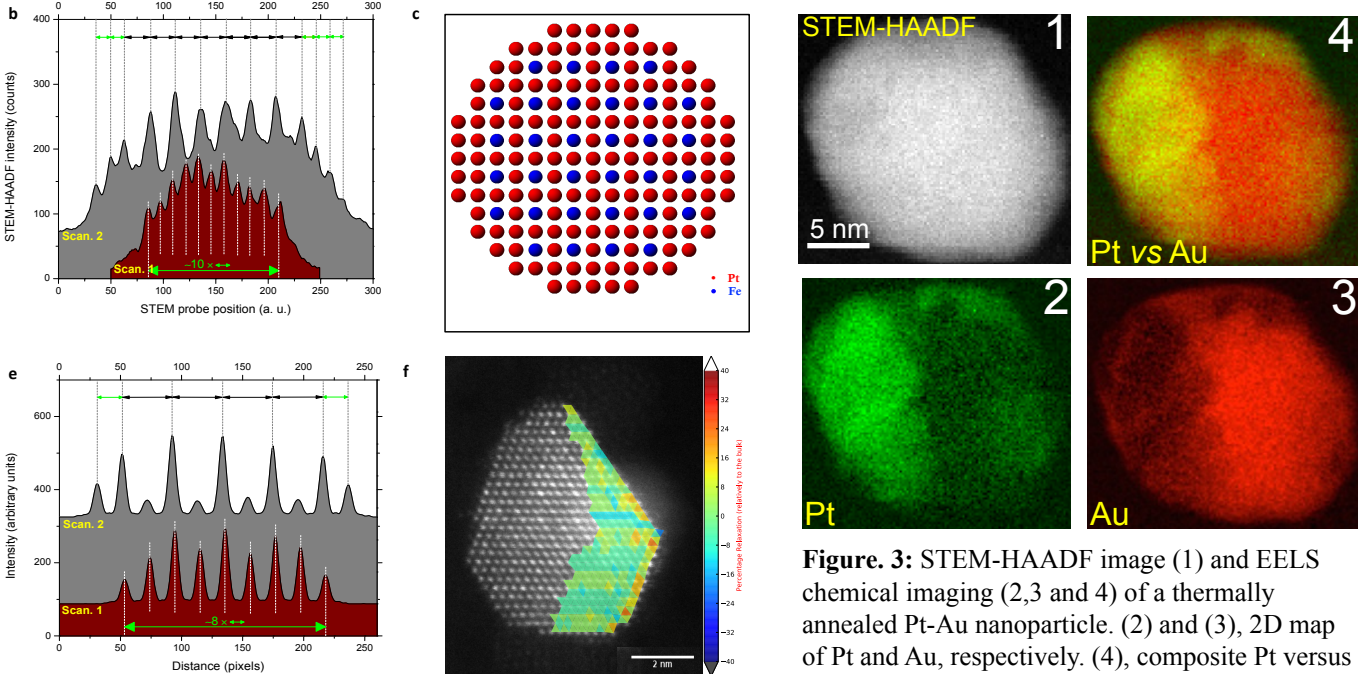

Figure. 3: STEM-HAADF image (1) and EELS chemical imaging (2,3 and 4$)$ of a thermally annealed Pt-Au nanoparticle. (2) and (3), 2D map of Pt and $\mathrm{Au}$, respectively. (4), composite Pt versus Au map. Clearly, these maps evidence a phase separation between $\mathrm{Pt}$ and $\mathrm{Au}$, corroborating with the immiscibility gap observed in the bulk phase diagram.
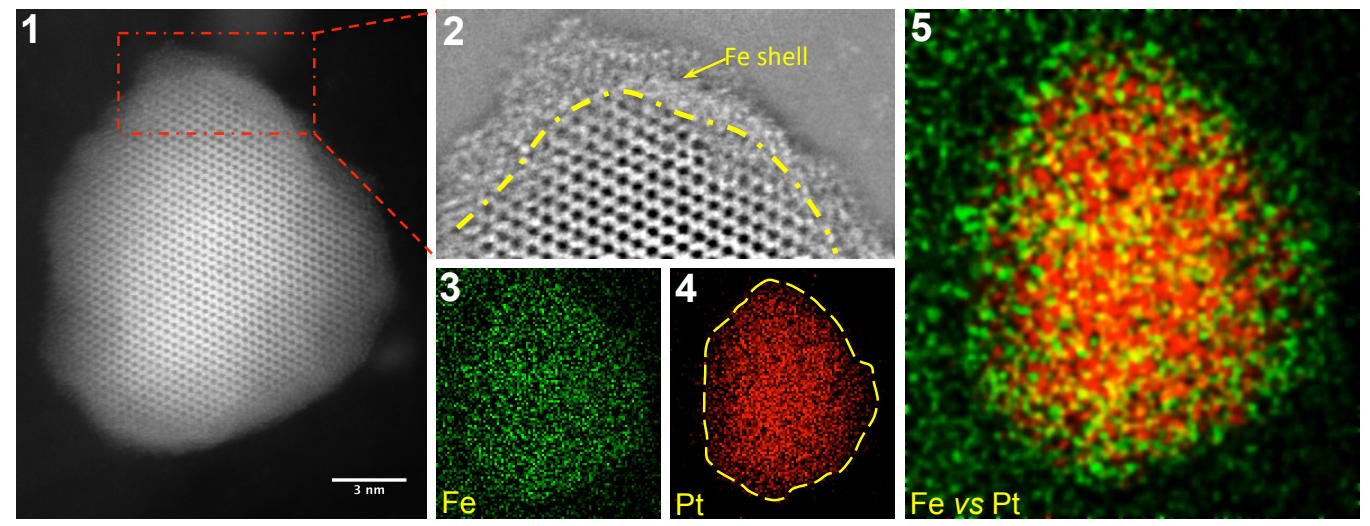

Figure. 2: (1), STEM-HAADF of a thermally annealed Pt-Fe particle. (2), Band-pass filtered image of a selected region from (1) (marked in red). (3) and (4), 2D EELS map of Fe and Pt, respectively. (5), the composite Fe versus Pt map 